\title{
Lattice Results for the QCD Phase Transition
}

\author{
Tereza Mendes \\ Instituto de Física de São Carlos, Universidade de São Paulo, \\ Caixa Postal 369, 13560-970, São Carlos, SP, Brazil
}

Received on 11 September, 2006

\begin{abstract}
We discuss recent results for the phase transition in finite-temperature QCD from numerical (Monte Carlo) simulations of the lattice-regularized theory. Emphasis is given to the case of two degenerate light-quark flavors. The order of the transition in this case, which could have cosmological implications, has not yet been established.

Keywords: QCD phase transition; Lattice formulation; Numerical simulations; Universality; Scaling
\end{abstract}

\section{INTRODUCTION}

Although we are still trying to understand how confinement arises in QCD, we know that under normal conditions the running coupling is large at low energies, causing quark and gluons to be confined. However, when exposed to extreme conditions such as very high temperatures or densities, quarks are forced to stay at very short distances from one another and there is a transition to a deconfined, quark-gluonplasma phase. This transition is present both at high temperature and at high density, implying a phase diagram where the hadronic phase exists only near the origin of the plane defined by the temperature and chemical-potential axes. Of course, one wants to know the location of the transition in order to study properties of the new phase of matter, but it is also interesting to determine the nature of this transition. In particular, it is important to establish if the transition is a strong one, of first order, involving discontinuity in the order parameter, or if it is such that the two phases are connected smoothly. This may have consequences for the understanding of the cosmological QCD phase transition, which occurred a few microseconds after the big bang and formed the hadrons we observe today. In this case the transition lies closer to the temperature axis and its nature is of direct importance to determine the types of cosmological relics that can be associated with it. In particular, a first-order transition would very likely be associated with the formation of cold dark matter clumps [1,2]. The nature of the transition must be also taken into account at relativistic heavy-ion collision experiments. An additional requirement in this case is the description of dynamic effects [3].

The task of studying the QCD phase transition theoretically must be carried out by nonperturbative methods and a natural choice is to consider the lattice regularization as a formulation of the theory. In fact, lattice-QCD simulations allow a nonperturbative description of the phase transition in hadronic matter at high temperatures and there has been some recent progress in the description of the transition also in the case of finite density [4]. In the case of the finite-temperature transition, there is a qualitative difference when dealing with the fullQCD case (i.e. considering dynamic quarks) or with the socalled quenched case, in which the gluonic effects are taken into account but sea quarks are taken to be infinitely massive. For the quenched case one studies the deconfining transition itself, by means of the order parameter given by the Polyakov loop. The transition in this case is of first order. In the fullQCD case there is no equivalent order parameter for the deconfinement transition and one must consider the chiral phase transition. This transition occurs when the chiral symmetry - exact in the limit of zero quark masses and spontaneously broken at low temperatures - is restored at high temperature. The case of two dynamic quarks, i.e. considering dynamic effects of only two degenerate light-quark flavors, corresponding to the up and down quarks, is particularly interesting. In this case, if the transition is of second order, one would expect to observe universal critical scaling in the class of the $3 d O(4)$ continuous-spin model [5, 6]. Also, in the continuum limit, simulations using different discretizations for the fermion fields should give the same results. The fact that the critical behavior should be in the universality class of a spin model can be precisely checked, since the nonperturbative behavior for these models can be obtained with Monte Carlo simulations by so-called global methods, which avoid the critical slowing-down present in QCD simulations [7,8].

The determination of the correct nature of the transition in the two-flavor case is one of the present challenges of lattice QCD, as pointed out by Wilczek in [9]. This prediction has been investigated numerically by lattice simulations for over ten years, yet there is still no agreement about the order of the transition or about its scaling properties $[4,10]$. More precisely, the predicted $O(4)$ scaling has been observed in the Wilson-fermion case [11], but not in the staggered-fermion case, believed to be the appropriate formulation for studies of the chiral region. In this case, extensive numerical studies and scaling tests have been done by the Bielefeld [12], JLQCD [13] and MILC [14] groups. It was found that the chiral-susceptibility peaks scale reasonably well with the predicted exponents, but no agreement is seen in a comparison with the $O(4)$ scaling function. At the same time, some recent numerical studies with staggered fermions claim that the deconfining transition may be of first order $[15,16]$.

In $[17,18]$ a simple method was introduced to obtain a uniquely defined normalization of the QCD data, allowing an unambiguous comparison to the (normalized) $O(4)$ scaling function. The analysis showed a surprisingly better agreement for the larger values of the quark masses. Let us note that in previous scaling tests the comparison had been done up to a (non-universal) normalization of the data and a match to the scaling function was tried by fitting it to the data points with the smallest masses. One interpretation of this result is that 
data at smaller masses (closer to the physical values) suffer more strongly from systematic errors in the simulations. In fact, larger quark masses are much easier to simulate, allowing greater control over errors and more reliable results. Here we present a preliminary study at a rather large mass value ( $m_{q}=0.075$ in lattice units), using staggered fermions and the MILC code. We consider the standard action and temporal lattice extent $N_{\tau}=4$, as in the studies mentioned above.

\section{SCALING TESTS}

The behavior of systems around a second-order phase transition (or critical point) may show striking similarities for systems that would otherwise seem completely different. In fact, it is possible to divide systems into so-called universality classes, in such a way that each class will have, e.g., the same critical exponents around the transition. Typical exponents are

$$
\begin{aligned}
M_{h=0, t \rightarrow 0^{-}} & \rightarrow|t|^{\beta}, \\
\chi_{h=0, t \rightarrow 0} & \rightarrow|t|^{-\gamma}, \\
M_{t=0, h \rightarrow 0} & \rightarrow h^{1 / \delta},
\end{aligned}
$$

where $M$ is the order parameter - e.g. the magnetization for a spin system $-\chi$ is the corresponding susceptibility and

$$
\begin{aligned}
t & =\left(T-T_{c}\right) / T_{0}, \\
h & =H / H_{0}
\end{aligned}
$$

are the reduced temperature and magnetic field, respectively.

Thus, in principle, one may compare the critical exponents for different systems to check if they belong to the same universality class. In practice, however, the critical exponents may vary little from one class to the other and in order to carry out the comparison one would need to have a very precise determination of the exponents, which is not yet feasible in the QCD case.

A more general comparison is obtained through the scaling functions for both systems. This comparison allows a more conclusive test, and can be applied for cases where the critical exponents cannot be established with great accuracy. In this case we may assume the exponents for a given class and compare the behavior of the whole critical region for one system to the known scaling curve for the proposed universality class. The scaling Ansatz is written for the free energy $F_{s}$ in the critical region as

$$
F_{s}(t, h)=b^{-d} F_{s}\left(b^{y_{t}} t, b^{y_{h}} h\right),
$$

where $b$ is a rescaling factor, $d$ is the dimension and $y_{t}, y_{h}$ are related to the usual critical exponents: $\beta, \gamma, \delta$ mentioned above. The scaling Ansatz implies that the order parameter must be described by a universal function

$$
M / h^{1 / \delta}=f_{M}\left(t / h^{1 / \beta \delta}\right) .
$$

The statement that the function $f_{M}$ is universal means that once the non-universal normalization constants $T_{0}$ and $H_{0}$ are determined for a given system in the universality class, the order parameter $M$ scales according to the scaling function $f_{M}$ for all systems in this class. As said above, the comparison of (normalized) scaling functions between two systems is a more general test of universality, especially in the case of the QCD phase transition.

A further difficulty in studying the critical behavior at the QCD phase transition is the impossibility of considering the critical point directly, since that would correspond to having zero quark mass, or zero magnetic field $H$ in the language of the spin models above. In order to check scaling with critical exponents of a given class, or to determine the normalization constants $T_{0}$ and $H_{0}$ for systems where a study at $H=0$ is not possible, it is important to determine the pseudo-critical line, defined by the points where the susceptibility $\chi$ shows a (finite) peak. This corresponds to the rounding of the divergence that would be observed for $H=0, T=T_{c}$. The susceptibility scales as

$$
\chi=\partial M / \partial H=\left(1 / H_{0}\right) h^{1 / \delta-1} f_{\chi}\left(t / h^{1 / \beta}\right),
$$

where $f_{\chi}$ is a universal function related to $f_{M}$. At each fixed $h$ the peak in $\chi$ is given by

$$
\begin{aligned}
t_{p} & =z_{p} h^{1 / \beta \delta}, \\
M_{p} & =h^{1 / \delta} f_{M}\left(z_{p}\right), \\
H_{0} \chi_{p} & =h^{1 / \delta-1} f_{\chi}\left(z_{p}\right) .
\end{aligned}
$$

Thus, the behavior along the pseudo-critical line is determined by the universal constants $z_{p}, f_{M}\left(z_{p}\right), f_{\chi}\left(z_{p}\right)$. Critical exponents, the scaling function $f_{M}$ and the universal constants above are well-known for the $3 d O(4)$ model [21-23].

Note that one may also consider the comparison for finitesize-scaling functions, since they are also universal and have the advantage of being valid for finite values of $L$, the linear size of the system. Such functions were studied for the $3 d$ $O(4)$ model in [22].

\section{COMPARISON OF QCD DATA WITH THE PREDICTED SCALING FUNCTION}

We now turn to the comparison of the two-flavor QCD data in the critical region (in the case of small but nonzero quark mass) to the predicted scaling properties of the $3 d O(4)$ spin model. As mentioned in the Introduction, we consider the chiral phase transition, since there is no clear order parameter for the deconfinement transition in the case of full QCD. The order parameter for the chiral transition is given by the so-called chiral condensate $\langle\bar{\psi} \psi\rangle$, where $\psi$ is a combination of the quark fields entering the QCD Lagrangian [6]. The analogue of the magnetic field is the quark mass $m_{q}$, and (on the lattice) the reduced temperature is proportional to

$$
6 / g^{2}-6 / g_{c}^{2}(0)
$$

where $g$ is the lattice bare coupling and $g_{c}^{2}(0)$ is its extrapolated critical value. Therefore, referring to the pseudo-critical 


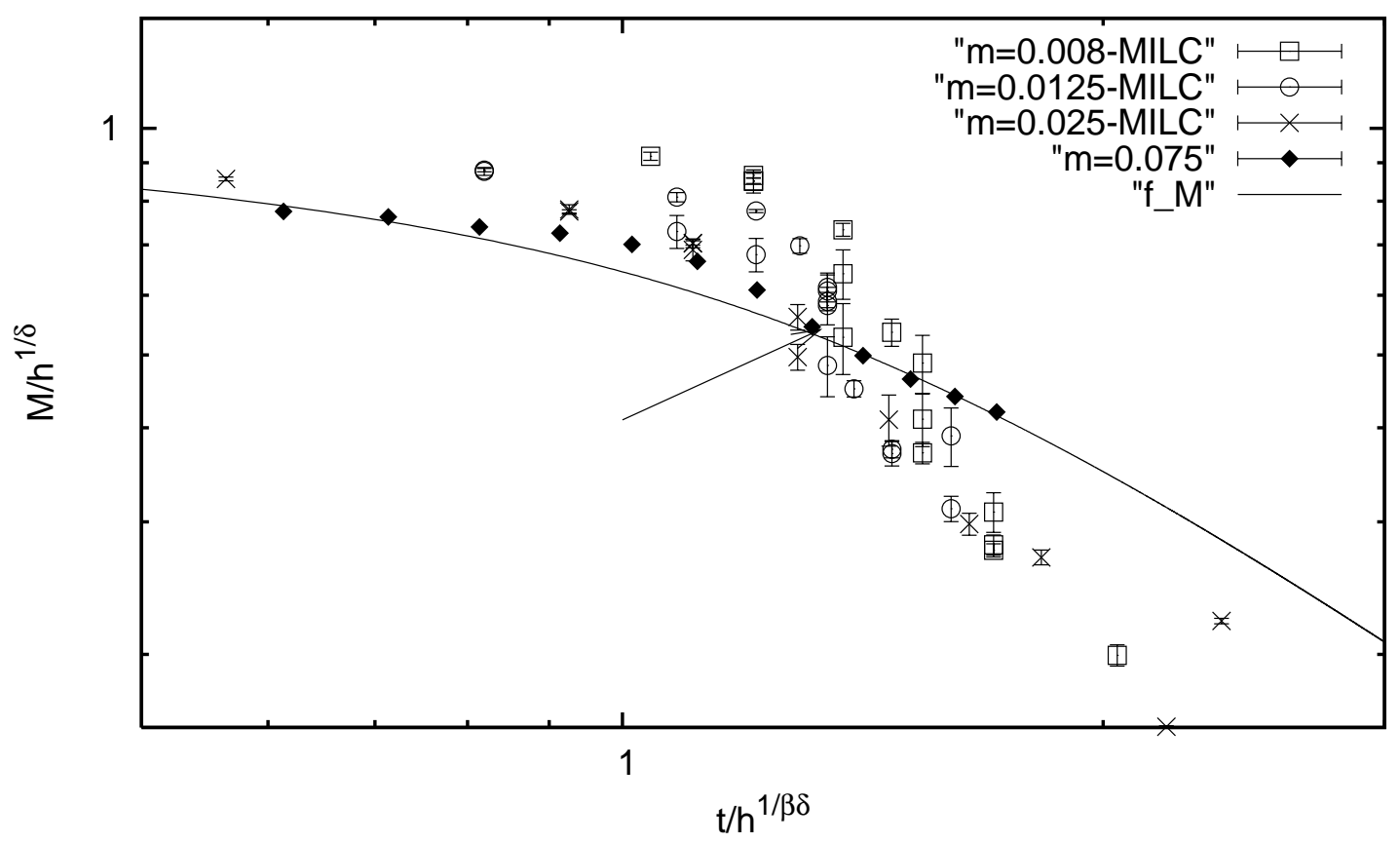

FIG. 1: Comparison of QCD (staggered) data to the $O(4)$ scaling function. For clarity, we do not show the data around the pseudo-critical point (indicated by the arrow), which were used to determine the normalization of the remaining data points.

line described in the previous section, the chiral susceptibility peaks at

$$
t_{p} \sim m_{q}^{1 / \beta \delta}
$$

As mentioned in the Introduction, previous results from lattice-QCD simulations in the two-flavor case show good scaling (with the predicted exponents) only along the pseudocritical line, which is given by the peaks of the chiral susceptibility. It should be clear from the discussion in the above sections that this is not a sufficient test to prove that the transition is second order, especially if no agreement is seen when comparing the data to the scaling function. As described in $[17,18]$, we use the observed scaling along the pseudo-critical line and the universal quantities $z_{p}, f_{M}\left(z_{p}\right)$ from the $O(4)$ model to determine the normalization constants $H_{0}, T_{0}$ for the QCD data. This allows an unambiguous comparison of the data to the scaling function $f_{M}$. More precisely, we note that in previous analyses [14] the normalization constants were tentatively adjusted by shifting the $O(4)$ curve so as to get a rough agreement with the data at smaller quark masses, since these are closer to the chiral limit. The problem is that the lighter masses are also more subject to the presence of systematic errors in the simulations. In this case the overall agreement was rather poor, indicating that there were strong systematic effets or that the transition is not in the predicted universality class. Here we fix the constants as described in Section II, following the behavior along the pseudo-critical line. In this way no value of the quark mass is priviledged and the comparison is unambiguous.

Our comparison is shown in Fig. 1 below. The pseudocritical line corresponds to a point in this plot and is marked with an arrow. For clarity we do not show the data (from the Bielefeld and JLQCD collaborations) obtained directly at the pseudo-critical point. These are slightly scattered around $z_{p}$ but show good scaling within errors.

We see relatively good scaling in the pseudo-critical region, i.e. around $\left[z_{p}, f_{M}\left(z_{p}\right)\right]$, as expected. Away from this region most MILC points are several standard deviations away from the predicted curve. These data are given for three values of the quark mass in lattice units: $0.008,0.0125$ and 0.025 . Note that the points with larger mass come closer to the curve. In particular, we can see that the new data at $m_{q}=0.075$ show sensibly better scaling, especially for larger temperatures, where previously the scaling seemed unlikely. The good agreement of these data with the $O(4)$ scaling function motivates a careful study of systematic errors for smaller masses. A possible source of such errors are finite-size corrections, which would be stronger for smaller masses, since then the lattice side may not be large enough to "contain" the physical particle. Put differently, finite-size effects are expected when the correlation length (in lattice units) associated with a particle is comparable to or larger than the lattice side. Of course, this is more likely to occur for a lighter particle.

\section{FINITE-SIZE EFFECTS}

In addition to the infinite-volume scaling laws mentioned above, we may also consider finite-size-scaling functions. In fact, the scaling Ansatz in Eq. 6 also implies

$$
M=L^{-\beta / v} Q_{z}\left(h L^{\beta \delta / v}\right)
$$


where $L$ is the linear size of the system and we consider fixed values of the ratio $t / h^{1 / \beta \delta} \equiv z$ (e.g. $z=0$ as in the critical isotherm, or $z_{p}$ as along the pseudo-critical line). Thus, $M$ can be described by a universal finite-size-scaling (FSS) function of one variable. We note that in order to recover the infinitevolume expression $M=h^{1 / \delta} f_{M}(z)$ as $L \rightarrow \infty$, we must have $Q_{z}(u) \rightarrow f_{M}(z) u^{1 / \delta}$ for large $u$. Thus, in this limit, the FSS functions are given simply in terms of the scaling function $f_{M}(z)$. Working with the FSS functions $Q_{z}$ instead of the infinite-volume scaling function $f_{M}$ has the disadvantage that one must consider $z$ fixed (thus restricting the regions to be compared in parameter space) but the advantage that a comparison can be made already at finite values of $L$.

A finite-size-scaling analysis as described above was carried out in [17], but it was found that the QCD data show good (finite-size) scaling only along the pseudo-critical line.

\section{CONCLUSIONS}

Understanding the nature of the chiral phase transition in two-flavor QCD has proven to be a challenging task. The prediction of a second-order transition with critical behavior in the universality class of the $O(4)$ spin model is not verified for staggered fermions of small masses, although it can be shown (by an unambiguous normalization of the data) that better scaling is obtained for the existing data at larger (unphysical) masses. The fact that data for heavier quarks would show such good scaling was not expected, since the normal- ization of the data for comparison with the scaling curve did not priviledge any particular values of the quark mass. This suggests that the lack of scaling at small masses observed so far may be due to systematic effects, which could be due to finite-size corrections or to uncontrolled errors in the hybrid Monte Carlo algorithm used for updating the configurations. Both these sources of errors would be more significant for the case of smaller masses. As discussed above in Section IV above, the deviations observed are most likely not due to finite-size corrections and we thus argue that the deviations from $O(4)$ scaling at smaller masses may come from systematic errors in the simulation, probably related to the use of the $\mathrm{R}$ algorithm for the simulations [24]. Note that, contrary to what happens for the quenched-QCD case, the algorithm used to update full-QCD configurations is not exact and should have its accuracy tested carefully for each different value of the quark mass used.

Let us also mention that a redefinition of the reduced temperature in terms of the physical temperature $T$ including a term in the quark mass $M$, as suggested in [16], improves the agreement with the scaling curve further, as has been recently shown in [20].

\section{ACKNOWLEDGEMENTS}

This work was supported by FAPESP (Grant 00/05047-5). Partial support from CNPq is also acknowledged.
[1] D. J. Schwarz, Annalen Phys. 12, 220 (2003).

[2] M. Hindmarsh and O. Philipsen, Phys. Rev. D 71, 087302 (2005).

[3] Y. Hama, T. Kodama, and O. J. Socolowski, Braz. J. Phys. 35, 24 (2005)

[4] O. Philipsen, PoS LAT2005, 016 (2005).

[5] R. Pisarski, and F. Wilczek, Phys. Rev., D 29, 338 (1984).

[6] K. Rajagopal and F. Wilczek, Nucl. Phys. B 399, 395 (1993).

[7] U. Wolff, Phys. Rev. Lett. 62, 361 (1989).

[8] T. Mendes, A. Pelissetto, and A. D. Sokal, Nucl. Phys. B 477, 203 (1996).

[9] F. Wilczek, Nucl. Phys. Proc. Suppl. 119, 3 (2003).

[10] S.D. Katz, Nucl. Phys. Proc. Suppl., B 129, 60 (2004).

[11] Y. Iwasaki, K. Kanaya, S. Kaya, and T. Yoshié, Phys. Rev. Lett., 78, 179 (1997).

[12] F. Karsch, and E. Laermann, Phys. Rev., D 50, 6954 (1994).

[13] S. Aoki et al. (JLQCD Collaboration), Phys. Rev., D 57, 3910 (1998).
[14] C. Bernard et al. (MILC Collaboration), Phys. Rev., D 61, 054503 (2000).

[15] P. Cea, L. Cosmai, and M. D'Elia, JHEP, 0402, 018 (2004).

[16] M. D’Elia, A. Di Giacomo, and C. Pica, Phys. Rev. D 72, 114510 (2005).

[17] T. Mendes, in Statistical QCD 2001, edited by F. Karsch and H. Satz. (Elsevier, Amsterdam, 2002), P29, hep-lat/0111044

[18] T. Mendes, in Hadron Physics 2002, edited by C.A.Z. Vasconcellos et al. (World Scientific, Singapore, 2003), 227.

[19] T. Mendes and A. Cucchieri, AIP Conf. Proc. 739, 599 (2004).

[20] T. Mendes, AIP Conf. Proc. 756, 413 (2005).

[21] J. Engels, and T. Mendes, Nucl. Phys., B 572, 289 (2000).

[22] J. Engels, S. Holtmann, T. Mendes, and T. Schulze, Phys. Lett., B 514, 299 (2001).

[23] A. Cucchieri and T. Mendes, J. Phys. A 38, 4561 (2005).

[24] M. A. Clark, P. de Forcrand, and A. D. Kennedy, PoS LAT2005, 115 (2005) 